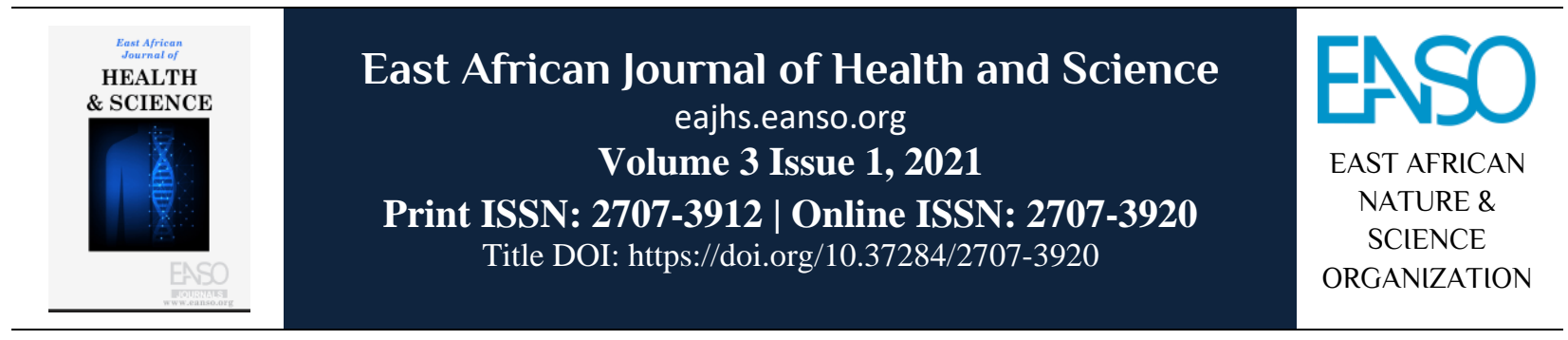

Original Article

\title{
Understanding the Role of Health Workers in the LF Elimination Program in Kenya: Challenges Faced and Suggestions for Improved Program Performance.
}

\author{
Bridget W. Kimani ${ }^{*}$, Dr. Lydiah Wanjiku Kibe, PhD ${ }^{1}$, Collins Okoyo ${ }^{1}$, Wyckliff P. Omondi ${ }^{2}$, Dr. \\ Hadley Matendechero Sultani ${ }^{2} \&$ Dr. Doris Wairimu Njomo, PhD ${ }^{1}$ \\ ${ }^{1}$ Eastern and Southern Africa Centre of International Parasite Control (ESACIPAC), Kenya Medical Research Institute \\ (KEMRI), Nairobi. P. O. Box 54840-00202, Nairobi, Kenya. \\ ${ }^{2}$ Division of Vector Borne and Neglected Tropical Diseases, Ministry of Health. P. O. Box 30016-00100, Nairobi, Kenya. \\ *Author for correspondence email: bridgetkimani@gmail.com
}

Article DOI: https://doi.org/10.37284/eajhs.3.1.393

\section{Date Published: ABSTRACT}

26 August 2021 The Kenyan Ministry of Health and its partners through the Division of VectorBorne and Neglected Tropical Diseases, is in charge of the Lymphatic Filariasis

Keywords: Mass Drug Administration programme. This is implemented through the national, county, and sub-county neglected tropical diseases coordinators. The current study Lymphatic sought to understand the roles, challenges faced and suggestions of how program filariasis, performance can be improved by the community health extension workers, county mass drug and sub-county neglected tropical diseases coordinators. Two wards of the administration, Kaloleni sub-county; Kilifi County were purposively selected. In 2015, Kaloleni challenges, and Kayafungo wards had a treatment coverage of 58\% and 54\% respectively; opportunities, $62 \%$ and $39 \%$ respectively in 2016, all below the recommended minimum Health Workers, treatment coverage of $65 \%$. Qualitative data was collected through sixteen inNeglected Tropical depth interviews with community health extension workers and two semi-

Diseases structured interviews with the county and sub-county neglected tropical diseases Coordinators. coordinators. Data were analysed by QSR NVIVO version 10 according to identified themes. The study results show the various roles in planning and implementation of the program include; supply chain management; health information education communication and records management; health workforce training and management, leadership and governance, and service delivery. Challenges faced included insufficiency of drugs supplied and information education communication materials, the inadequacy of community drug distributors selected and trained, poor facilitation for training and supervision of community drug distributors, limited duration of the mass drug administration,

126 This work is licensed under a Creative Commons Attribution 4.0 International License. 
and delayed reporting due to poor network coverage. The results of this study show that the community health extension workers, county and sub-county neglected tropical diseases coordinators are not fully involved in program leadership and governance, a role that is taken up at the national level. They should be involved in all the stages of the mass drug administration program to create ownership to improve the program performance.

\section{APA CITATION}

Kimani, B. W., Kibe, L. W., Okoyo, C., Omondi, W. P., Sultani, H. M., \& Njomo, D. W. (2021). Understanding the Role of Health Workers in the LF Elimination Program in Kenya: Challenges Faced and Suggestions for Improved Program Performance. East African Journal of Health and Science, 3(1), 126-144. https://doi.org/10.37284/eajhs.3.1.393.

\section{CHICAGO CITATION}

Kimani, Bridget W, Lydiah Wanjiku Kibe, Collins Okoyo, Wyckliff P. Omondi, Hadley Matendechero Sultani, and Doris Wairimu Njomo. 2021. "Understanding the Role of Health Workers in the LF Elimination Program in Kenya: Challenges Faced and Suggestions for Improved Program Performance". East African Journal of Health and Science 3 (1), 126-144. https://doi.org/10.37284/eajhs.3.1.393.

\section{HARVARD CITATION}

Kimani, B. W., Kibe, L. W., Okoyo, C., Omondi, W. P., Sultani, H. M., \& Njomo, D. W. (2021) "Understanding the Role of Health Workers in the LF Elimination Program in Kenya: Challenges Faced and Suggestions for Improved Program Performance", East African Journal of Health and Science, 3(1), pp. 126-144. doi: 10.37284/eajhs.3.1.393.

\section{IEEE CITATION}

B. W. Kimani, L. W. Kibe, C. Okoyo, W. P. Omondi, H. M. Sultani, \& D. W. Njomo, "Understanding the Role of Health Workers in the LF Elimination Program in Kenya: Challenges Faced and Suggestions for Improved Program Performance.”, EAJHS, vol. 3, no. 1, pp. 126-144, Aug. 2021.

\section{MLA CITATION}

Kimani, Bridget W, Lydiah Wanjiku Kibe, Collins Okoyo, Wyckliff P. Omondi, Hadley Matendechero Sultani, and Doris Wairimu Njomo. "Understanding the Role of Health Workers in the LF Elimination Program in Kenya: Challenges Faced and Suggestions for Improved Program Performance". East African Journal of Health and Science, Vol. 3, no. 1, Aug. 2021, pp. 126-144, doi:10.37284/eajhs.3.1.393.

\section{INTRODUCTION}

Lymphatic filariasis (LF) is a neglected tropical disease and that continues to be a major cause of morbidity and permanent disability in endemic populations [1,2]. Infection is caused by a mosquito-transmitted filarial worm. In 2018, 893 million people across 49 countries were living in areas at risk of $\mathrm{LF}$ and required preventive chemotherapy to stop the spread of infection [3].

The WHO in 2000, launched the Global Programme to Eliminate Lymphatic Filariasis (GPELF) as a public health problem in response to World Health Assembly resolution, WHA 50.29, which urged member states to initiate activities to eliminate lymphatic filariasis (LF) [3]. Recently in the year 2020, WHO launched a roadmap for neglected tropical diseases 2021-2030 aimed at ending the neglect to attain sustainable development goals. This was as a result of not meeting all the targets that had been set for 2020 in the earlier roadmap [4]. The GPELF strategy has been to promote large scale single-dose community-wide mass drug administration (MDA) of antifilarial tablets to entire at-risk populations aged two years and above [5]. This is to ensure the reduction of microfilariae levels in human populations in order to interrupt the transmission cycle between mosquitoes and humans. The minimum recommended annual MDA for LF coverage by the WHO for 4-6 consecutive years is $>65 \%$ of the total population [6]. However, this is usually dependent on the microfilariae baseline prevalence in the population at risk and other factors determining transmission [7].

In 2007, WHO formulated a health systems framework that described health systems in terms of six building blocks which include: service delivery; health workforce; information; medical products, vaccines, and technologies; financing and leadership, and governance. Some cross-cutting 
components, such as leadership and governance and health information systems, provide the basis for the overall policy and regulation of all the other health system blocks. Key input components to the health system include specifically, financing and the health workforce. A third group, namely medical products and technologies and service delivery, reflects the immediate outputs of the health system, i.e., the availability and distribution of care [8].

Studies indicate the WHO health system framework is instrumental in strengthening the overall health system and uses it as a catalyst for achieving global health targets such as the Sustainable Development Goals (SDGs). Unlike other health system strengthening strategies that are disease-specific [9] or narrow [10-12], the WHO's health system framework intends to improve the overall health in a responsive, financially fair, and most efficient way [8]. Evidence revealed that the framework helps to assess in-country healthcare performances [13], interactions between health reforms and country health systems [14], implications of health sector reforms [15], and the status of health facilities [16] and specific health problems [17].

Many countries have implemented MDA for LF campaigns, successfully reducing the prevalence levels of the microfilariae in endemic populations and between the year 2000 and 2015, the WHO reported that more than 6.2 billion doses of treatment were administered to more than 830 million people in 64 endemic countries, reducing the transmission risk by 45\% [18]. Despite these positive global achievements in treatment provision, MDA for LF programmes continues to face numerous challenges that result in low treatment coverage levels and non-compliance by the communities [19-25]. Programmatic challenges include; sustaining timely distributions of drugs, establishing accurate monitoring and evaluation systems by the communities, increasing involvement of the local communities, and engaging in effective advocacy for continued MDA for LF support [7]. It has been noted that MDA for LF programmes seems to focus more on tablet distribution than on the major implementation questions such as health education, side effects, morbidity management, and logistics [24]. Similarly, there are also reports that problems of low compliance to MDA for LF are more provider- initiated than by individual recipients' perceptions and practices. Several reports have highlighted implementation challenges in MDA for LF programmes [26-29].

In Kenya, LF is endemic in six counties of the Coastal region, where ecological factors are suitable for its transmission [18]. The Ministry of Health through the Division of Vector-Borne and Neglected Tropical Diseases (DVBNTD) launched the LF elimination programme in 2002 in Kilifi District as the first implementation unit (IU). In 2003, the Programme was scaled up to include Malindi and Kwale districts and in 2005 and 2008, the 3 districts received additional rounds of MDA but due to technical and administrative challenges, sustained annual MDA was not possible. In 2015 the program was re-started and has since achieved $100 \%$ geographical coverage [18]. In 2018, WHO published a new guideline on alternative MDA regimens and recommended a 3-drug regimen; ivermectin, diethylcarbamazine, and albendazole (IDA) to accelerate the elimination of LF as a public health problem. In November 2018, the Kenyan Ministry of Health (MOH) launched the piloting of this new drug regimen [19].

The Kenyan Ministry of Health and its partners through the DVBNTDs implements the LF MDA programme in line with the Community Health Strategy (CHS) launched in 2006 [20]. The National NTDs coordinator implements the LF MDA activities through the county and sub-county NTDs coordinators who are public health officers selected by the national NTDs programme together with the county health management team (CHMT) and subcounty health management team (SCHMT). The CNTDs and SCNTDs coordinators are in charge of all the NTDs activities at both county and subcounty levels and are involved in planning, coordination, drug storage and distribution, mobilization, sensitization, and training of county and sub-county health management teams; drug administration; management of severe adverse events; supervision, and record-keeping where they submit the collated data to the national NTDs coordinator. In the CHS, linkages at the county, subcounty, health facilities, and community levels, the health facility in-charges CHMT, CHEWs, community health workers (CHWs), village elders and chiefs, and other extension workers, are the 
sinews that bind these structures and enable sustained community leadership in addressing health problems, through the formation of linkage committees at all these levels [20].

The CHEWs who are selected by the sub-county public health officers (SCPHO) are in-charge of health facilities. They are charged with training, drug distribution, and supervision of community drug distributors (CDDs), who distribute drugs to allocated households in the villages. They are also involved in community sensitization and mobilisation through the local administration, management of SAEs and record-keeping and through the reverse cascade [20] to ensure that all the recorded data and any remaining drugs are surrendered to the sub-county PHOs who then ensure that they are returned to the CNTDs coordinator through the SCNTDs coordinators.

The current study sought to understand the roles played by the CHEWs, CNTDs, and SCNTDs coordinators, challenges faced, and suggestions for improved program performance.

\section{MATERIALS AND METHODS}

\section{Study Area}

This study was conducted in Kaloleni sub-county in Kilifi County. It is made up of four administrative units: Kaloleni, Kayafungo, Mwanamwinga, and Mariakani [21] It has a population of 159,739 with Kaloleni and Kayafungo having a population of 41,689 and 22,250 respectively [22]. Farming and business form the main economic activities in the area [23].

\section{Study Design and Setting}

The data being presented in the current paper was qualitative and was collected through separate indepth interviews (IDIs) conducted with 16 health workers and 2 NTDs coordinators. This was part of a larger quasi-experimental study with a preintervention and a post-intervention phase that utilized quantitative and qualitative methods for data collection. Based on the LF MDA Programme data from $\mathrm{MOH}$, Kenya, Kaloleni sub-county was purposively selected owing to its low, $56 \%$, and $50.5 \%$ treatment coverage in 2015 and 2016 respectively. Kaloleni and Kayafungo wards in
Kaloleni sub-county were then purposively selected for the study. In 2015, Kaloleni ward had a treatment coverage of 58\% and Kayafungo, 54\%, and in 2016, Kaloleni had 62\% and Kayafungo 39\% all below the recommended minimum treatment coverage of $65 \%$.

\section{Participant selection}

With the support of the area chiefs, a total of 6 villages in Kaloleni and Kayafungo wards were purposively selected as they were considered to have had implementation challenges. The selected villages were Town Centre and Vishakani for Kaloleni ward and Mirihi ya Kirao, Gogoraruhe, Gandini A, and Gandini B for Kayafungo ward. Due to their specific roles and responsibilities in the LF MDA, the CHEWs, county, and sub-county NTDs coordinators were purposively selected to participate in this study.

\section{Data Collection}

With the aid of an interview guide, the data was collected using the English language and moderation was done by the KEMRI social scientists assisted by eight trained field assistants. The field assistants were trained for three days prior to the start of the data collection exercise to enable them to understand the scope of the study. They were trained on lymphatic filariasis which included; pathophysiology, symptoms, diagnosis, complications, prevention, treatment, and management. They were also trained on qualitative data collection methods which included; data collection techniques, proper use of voice recorders, and data transcription. Data collection was conducted among sixteen CHEWs using IDIs guides and one CNTDs and one SCNTDs coordinator using SSIs. These interviews covered issues such as their role and views on the LF MDA campaign design, the specific activities, and practices they employed in their work and their perceived outcomes, challenges they faced in the implementation of the LF MDA campaign, insights about how they were received by members of the community during the drug distribution process and their suggestions towards improving the implementation of the LF MDA. Standard procedures including maintaining a neutral stance, probing and allowing the respondents to express 
themselves without asking leading questions, asking general questions before specific questions, and varying questions wording to avoid seeming repetitive were adhered to [24]. Each IDI took 50 to 60 minutes at a quiet and private venue determined by both the participant and field assistant, to ensure privacy. Notes were taken during the IDIs and voice recorders were used to record all the information. Permission to record was obtained from the participants during consenting [25]. Information on the roles played by the CHEWs, CNTDs, and SCNTDs coordinators, challenges encountered and their opinion of how the program performance can be improved was elicited.

\section{Data Management}

The data management process started with a collection of information gathered through field notes and audio recordings of the IDIs. The two data sets were compared and the recorded data was transcribed verbatim. The transcriptions underwent quality assurance reviews to ensure accuracy throughout the process and any discrepancies were discussed among the investigators. All the written notes were stored in lockable secure cabinets and the soft copies were stored in password-protected computers. Access to the hardcopies and transcripts was only upon authorization by the Principal Investigator.

\section{Data Analysis}

Data from the IDIs were analysed using the thematic analysis technique, in NVIVO 10 software by QSR international. This technique enables the identification and exploration of themes within the coded data [25]. A code list was developed comprising broad themes which were iteratively agreed upon among the research team members after a preliminary reading of the IDI transcripts and later modified to accommodate emergent themes. The textual data was coded into selected themes and a master sheet analysis was carried out, giving all the responses a theme. Manual analysis was further conducted according to study themes that were determined prior to the analyses. A thematic framework approach was adopted based on themes around the WHOs health systems framework: (i) service delivery;(ii) health workforce; (iii) information; (iv) medical products, vaccines, and technologies; (v) financing; and (vi) leadership and governance. The themes identified were; Leadership and Governance, Health Workforce Training and Management, Supply Chain Management, Service Delivery, Health Information Education Communication (IEC), and Records Management. The analysed data was presented in text form.

\section{Ethical considerations}

This study was reviewed and approved by the Kenya Medical Research Institute (KEMRI), Scientific and Ethics Review Unit (SERU No. 3666). The study participants who were all above 18 years of age provided written informed consent, where they also agreed to have the IDIs audio recorded. Permission to carry out the study was obtained from the respective authorities of Kilifi County. An information sheet was provided to the NTD coordinators and CHEWs invited to participate in the study in the English language.

\section{RESULTS}

\section{Background characteristics of the study participants}

\section{The NTDs Coordinators}

The NTDs coordinators are designated public health officers one male $(n=1)$ and one female $(n=1)$, each heading the county and the sub-county respectively. A total of two SSIs $(n=2)$ were carried out. These interviews covered issues such as their role, challenges encountered, and views on how the LF MDA campaign can be improved for better performance.

\section{The CHEWs}

A total of sixteen CHEWs participated in the IDIs. The gender distribution was half $(n=8)$ male and half female. These interviews covered issues on their role and views on the LF MDA campaign design, the specific activities, and practices they employed in their work and their perceived outcomes, challenges they faced in the implementation of the LF MDA campaign, and their suggestions towards improving the implementation of the LF MDA. 


\section{Planning and Coordination of the LF MDA}

The LF, NTDs county, and sub-county coordinators are directly involved in planning, coordination, and implementation of the LF MDA activities, in line with the WHO guidelines. These include but are not limited to sub-county meetings with stakeholders involved in the program, distribution and storage of the drugs, training, community mobilisation, sensitisation, and control of lymphatic filariasis. The CNTDs and SCNTDs coordinators described the activities that they are involved in during planning and coordination as:

"It involves coordinating sub-county meetings with other stakeholders who are involved in this program such as Evidence Action and the Ministry of Health at National Level" (IDICNTD).

"There was another round of distribution where I participated in planning for the distribution especially logistical activities at the Kilifi county. I also participated in choosing the sentinel sites in Magarini, Kayafungo, and Jaribuni. The survey was conducted before the drug distribution" (IDI-SCNTD).

During the planning and coordination of LF MDA, one of the major challenges was the number of CDDs. The ratio of CDDs allocated to households (HH) and or area covered was very few. The CNTDs coordinator and a CHEW from Kaloleni (KLN) stated that:

"500 HH in urban areas is achievable as they a $\mathrm{HH}$ are located close to each other but in the rural areas, it is challenging because houses are sparsely populated and one is required to walk long distances to reach the households but this walking time is not factored in" (IDI-CNTD).

"The CDDS were not many comparing to the area they covered" (IDI-KLN-HW-002).

Lack of involvement of the SCHMT was another challenge that was experienced. The SCNTD coordinator stated that:

"The sub-county health management team is not directly involved. They feel like they have been left out. They participate in the planning meetings but the actual implementation limits the number of people thus not owning the process e.g., nurses, clinical officers" (IDI-SC-NTD).

Another challenge experienced was the duration of the MDA process where the time allocated was not adequate to carry out all the required activities. A CHEW from Kayafungo (KYG) stated that:

"When this program starts it's usually like a marathon. In one week, everything is done. In that same week, the health workers are trained, the CDDs are also trained, the people are trained and the MDA is conducted which is not enough" (IDI-KYG-HW-002).

Transport was also another challenge faced by both the CHEWs and CDDs. A CHEW from Kaloleni stated that:

"The problem was transport for both CDDs and community health workers because bikes are few and at the end of the activity you have to pick the tallying sheets every day because the CDDs can't come to the office every day to bring them. I had to select a specific point where I can collect them. Also, we had a challenge during the follow-up" (ID-KLN-HW-001).

Despite the challenges experienced during planning and coordination, there were suggestions of how this can be improved to enhance the uptake. It was suggested that the involvement of various stakeholders was key so as to create ownership and sustainability of the program. The SCNTDs coordinator stated that:

"Involvement of the sub-county health management team to own the program and to ensure sustainability” (IDI-SC-NTD).

Another suggestion was to increase the number of CDDs. A CHEW from Kayafungo stated that:

"They should increase the CDDs because when you have many CDDs everybody in the village will get drugs" (IDI-KYG-HW-001).

There was a suggestion on improving transport and involving the county in funding in form of providing vehicles to the program to support the supervision process was also another suggestion. The SCNTDs coordinator stated that:

131 This work is licensed under a Creative Commons Attribution 4.0 International License. 
"To ensure effective supervision, the supervision team at the county and sub-county should have a vehicle assigned to the activity through the period - mobilization, training, and during the distribution period. Transport is given in monetary form and is not adequate. County to be more involved in funding to enable support during supervision (especially provision of vehicles)" (IDI-SC-NTD).

A CHEW from Kaloleni stated that:

"They should improve in terms of transport. Transport in the sense that the health workers easily pick the drugs from the sub-county pharmacy and also delivering to the CDDs in cases there is a shortage of drugs while the CDD is in the field. The CDDs should also be increased in number" (IDI-KLN-HW-002).

\section{Health Workforce Training and Supervision}

\section{Training}

Cascade training for integrated drug administration was carried out before the implementation of the LF MDA. The training sessions started at the regional level and cascaded down to the community level. Various cadres of people are trained at various levels. This training covers various components of the MDA such as what LF is, cause, transmission, prevention and control, advocacy, community sensitisation and mobilisation, drug collection, storage and distribution, documentation, and reporting. A two-day training is conducted targeting the county NTDs coordinator and county pharmacist; trainer of trainers (TOT). The county TOT then conducts a one-day training for the implementing units- sub-counties, comprising the sub-county PHO, NTDs coordinator, and health records information officer. The NTDs coordinators stated

that:

"The trainer of trainers (TOT) conducts a two days training targeting county-level staff and comprises of the county NTD coordinator and county pharmacist. The TOTs also train the implementing units (sub-counties) comprising of PHOs, NTD coordinator, and the health records information officer" (IDI-SC-NTD).
"I was directly involved in training. They are trained on the drugs to be used that is the two drugs DEC and ALB. They are also trained on the disease itself, transmission, and prevention. They are trained on advocacy and mobilization and also on how the drugs will be distributed" (IDI-CNTD).

Prior to their assignment of various roles, the health workers who comprise community health extension workers (CHEWs), undergo a one-day training conducted by the public health officers (PHOs) assigned to them. The CHEWs subsequently conduct a one-day training for the community drug distributors (CDDs). The CHEWs stated that:

"We were told the round of the Mass Drug Administration (MDA), it was the fourth round and we were basically given details on how it was supposed to be carried out, the percentage coverage that we were to get to and we were also shown how we were to train the CDDS on filling the tally sheets and how to take the record for the drugs" (IDI-KYG-HW-002).

"We started the introduction part the disease LF is all about then we discussed the causes of the disease and their prevention and importance of taking the drugs how to communicate with the community also how to tally. There are these sheets given to CDDs we used to do tallying and also make summary" (ID-KLN-HW-001).

When asked if they thought that there should be an addition of more content during training, the CHEWs felt that the content during training was adequate and felt that there was no need for additional information. A CHEW from Kayafungo stated that:

"It was not bad because it is a one-day event. It was mainly about the side effects of the drugs and how to administer the drugs according to age. So yes, it was adequate to undertake the assigned job” (IDI-KYG-HW-001).

Some challenges were expressed where some health workers felt that the one-day training was inadequate to undertake the job assigned to them for the programme. This was also echoed by the NTDs coordinators who felt that the time used for the training was inadequate. This was because some

132 | This work is licensed under a Creative Commons Attribution 4.0 International License. 
CHEWs had to travel from far and arrived late at the venue which led to the trainings starting late and ending early so as to ensure that there was enough time to travel back to their respective residences. Due to this, the training was hurried and not all the content was covered in-depth as expected. They stated that:

"One-day training is not enough maybe 2 days will be ok next time" (IDI-KYG-HW-006).

".... It is quite a challenge as the time allocated for training is not adequate to exhaustively cover all that is required plus the training materials are too many" (IDI-SC-NTD).

There were suggestions on increasing the number of days for training the CDDS, to ensure that trainers have enough time to cover the full curriculum. The CNTDs coordinator stated that:

"But the CDDs training has always been an issue because of the time allocated for the training. The training is meant to be one day but since the CDD have to travel to the venue, the trainings start late and end early to give the CDD time to travel back to their villages. This leaves like 5 hours for the training. This therefore, makes the training to be hurried as the trainers concentrate more on the drug distribution and reporting procedures. Most information about the disease is left out. It will be important to have the training allocated for two days. This will ensure coverage of the curriculum conclusively especially for newly recruited CDDs" (IDI-CNTD).

\section{Supervision}

Supervision was done right from the county to the community level. This was done during training, mobilisation, sensitisation, and drug distribution. The CHEWs supervised the CDDs during drug distribution. During supervision, they handled issues that arose such as drug supply in case they were not enough, and addressing any refusals in the community. A CHEW from Kayafungo stated that:

"It is supervising the CDDs, knowing that they are at the field and are giving out the drugs. At times I was going with them from one household to another seeing whether they have problems like refusals and such, then whenever they needed drugs, I went to where they were and supplied them with more drugs, then in case of anything they just call" (IDI-KYG-HW-002),

There were challenges that were experienced during supervision such as the vastness of the area to be covered, inadequate amount of time to carry out the supervision, and the ratio of CHEWs versus the CDDs to be supervised. The SCNTDs coordinator stated that:

"The number of CHEWs assigned to CDDs for supervision are few as the CDDs are very many. Support for transport and supervision is very limited to be able to do spot checks on the CDDs and $\mathrm{HH}$ to ensure that the drugs have been distributed. Spot Check-The time is inadequate as it is random thus unable to access all areas to be covered" (IDI-SC-NTD).

One of the suggestions for improving the supervision mechanism was, to increase the number of CHEWs. The SCNTDs coordinator stated that:

"Increase the number of CHEWs being utilized to ensure proper supervision of CDDs" (IDISC-NTD).

\section{Supply Chain Management}

\section{Drug Collection and Storage}

The drugs albendazole (ALB) and diethylcarbamazine (DEC) are procured nationally through Kenya Medical Supplies Agency (KEMSA). They are delivered to the county drug stores where they are received by the county pharmacist, who oversees their distribution to the sub-county pharmacist. From the sub-county stores, they are distributed to the health facilities. The CHEWs, who are assigned to be in charge of the CDDs by the public health officer, then collect and distribute them to the trained CDDs. The CNTDs coordinator from Kaloleni stated that:

"Drugs are bought and distributed through KEMSA. They are delivered to the county pharmacist and then to the sub-county. From here they are distributed to the facilities. The CDDs after training then collect the drugs 
according to the no of people, they have registered" (IDI-CNTD).

A CHEW from Kaloleni further stated that:

"They were collected from sub-county level, then brought down to the ward level then from there to the CHEWs. So, the CHEWs collected the drugs and took them to the training sites to the CDDs" (IDI-KLN-HW-003).

One of the challenges faced was the storage of the drugs during distribution. The CDDs were expected to take back any drugs that remained in their respective health facilities at the end of the day. A CHEW from Kayafungo stated that:

"The biggest challenge was on the CDDs how they stored the drugs because they couldn't bring them back by evening after distribution but basically those who were near the facility they could come back and store the drugs without difficulty" (IDI-KYG-HW-002).

Another challenge cited by the health workers was the shortage of drugs. The CDDs were issued with drugs based on the records on the household register which contained the number of household members. A CHEW from Kayafungo stated that:

"On the drugs, the drugs are not enough. At some point, you get a shortage and actually you have to like borrow from other people to supply your people which is not that convenient because the other person needs the same drugs" (IDIKYG-HW-002).

\section{SERVICE DELIVERY}

\section{Drug Distribution}

After training, each CDD collects the drugs according to the requirements, as entered in the village household registers. These are generated prior to the drug distribution process, which is done door to door by the CDDs where they utilize the directly observed treatment (DOT) mode of administration. A CHEW from Kaloleni stated that:

"I was making sure that I collect the drugs from the sub-county hospital, do the distribution to the $C D D s$, and before the distribution of drugs we also gave them registers to make sure that they fill the members who are eligible to get the drugs, and to add and subtract for those who died and those who have attained eligible age of receiving drugs. I had to make sure all the CDDs have received the drugs they require to give to the community. I also had to keep the communication with CDDs while in the field and even sometimes go to the field, to check if really the communities are receiving the drugs..." (IDI-KLN-HW-001).

The Vastness of the area to be covered was a common challenge amongst the NTDs coordinators and CHEWs. The number of households assigned to the CDDs was also a challenge depending on the area to be covered. They stated that:

"In some areas, CDDs were overwhelmed and did not reach all the households due to vastness of these areas. In some instances, we had to pull some CDDs to cover the households" (IDICNTD).

"The CDDs complained that the area that they were allocated were too big ..." (IDI-KLNHW-002).

"500 in urban areas is achievable as the HH are located close to each other but in the rural areas, it is challenging because houses are sparsely populated and one is required to walk long distances to reach the households but this walking time is not factored in. Thus, in rural areas, the target should be reduced to an achievable number of 300HH" (IDI-CNTD).

Another challenge that was experienced during the drug distribution process was that the length of the drug distribution was too short. This contributed to poor coverage as there was not enough time for the CDDs to revisit the missed households. The CNTDs coordinator stated that:

"Some households are not reached distribution and because the distribution period is short, the CDDs do not time for recall to missed households. A CDD is expected to reach $500 \mathrm{HH}$ in 3 days. Sometimes in urban areascosmopolitan, high literacy levels, the people especially those working in the market leave early and return late thus it is difficult to reach them" (IDI-CNTD).

134 | This work is licensed under a Creative Commons Attribution 4.0 International License. 
There were various suggestions and opinions on how the drug distribution could be improved. The length of the drug distribution was not sufficient and this contributed to poor coverage as there was not enough time for the CDDs to revisit the missed households. It was suggested that the number of days should be increased to allow for revisits. The CNTDs coordinator and a CHEW from Kaloleni stated that:

"Drug distribution period to be extended to one week. From Monday to Monday. This will give CDDs 3 days of active distribution and 4 days, passive distribution where those who missed the drugs during the distribution can access them from the CDDs homes" (IDI-CNTD).

"Maybe I say, the time for drug distribution is usually short so I would suggest this the time for drug distribution be increased, at least we distribute the drugs for one week or two weeks" (ID-KLN-HW-001).

The involvement of the local administration during drug distribution was one of the suggestions to enhance ownership by the community members. A CHEW from Kaloleni stated that:

'I have a suggestion that MDA is nice but to improve on it the community drug distributers there should be involvement of villages elder and nyumba kumi initiative elders, we should not only focus on CHVs' (IDI-KLN-HW-002).

It was suggested that the number of households assigned to a CDD be reconsidered especially in the rural areas where the houses are sparsely located as opposed to the urban areas where the houses are close to each other. The CNTDs coordinator stated that:

"500 in urban areas is achievable as the HH are located close to each other but in the rural areas, it is challenging because houses are sparsely populated and one is required to walk long distances to reach the households but this walking time is not factored in. Thus, in rural areas, the target should be reduced to an achievable number of $300 H$ H" (IDI-CNTD).

\section{Health Information Education Communication and Records Management}

\section{Mobilisation and Sensitisation for LF MDA}

Various stakeholders are involved during the mobilisation and sensitisation process prior to and during the LF MDA period. This is done right from the county up to the community level. This is done through meetings with different stakeholders, community barazas, door-to-door campaigns, radio advertisements and talk shows, IEC materials. At the county level, the NTD coordinator and county pharmacist sensitise the governor and county commissioner. At the sub-county level, the subcounty commissioner, chiefs and their assistant, incharges of health facilities are sensitized. Village barazas are organised to sensitize the community. The community is also sensitised by the CDDs during household registration. The SCNTDs coordinator stated that:

"At the sub-county - the sub-county commissioner, chiefs, assistant chiefs, incharges of health facilities are sensitized by the $M O H$, data records, and NTD sub-county coordinator. Village barazas are organized to sensitize the community on the drug distribution exercise and select drug distributors. This is the right procedure for selecting CDDs but in most cases, the CHVs are the ones who are recruited to be drug distributors" (IDI-SCNTD).

During sensitization, talk shows were held in the local radio broadcasting stations, so as to ensure wide coverage among the community members. The CNTDs coordinator stated that:

"We also had two radio stations talk show: Shungwaya broadcasting Services based in Kilifi and LULU FM based in Malindi. This was aimed at reaching a wide coverage with information about MDA and the importance of taking the drugs" (IDI-CNTD).

After training the health workers undertook community sensitisation and mobilisation activities. This was done before the actual treatment exercise, through community meetings (barazas), social gatherings, schools, through religious leaders, and door to door. The CHEWs did follow-ups a number of times to ensure that the majority of the

135 | This work is licensed under a Creative Commons Attribution 4.0 International License. 
community members had received the information. The CHEWs stated that:

".......Mobilize the community through maybe if the schools are still open, we go to schools and tell students, through the chiefs and other administrative and community gatekeepers all are told to help get the awareness to community members. And using the CHVs to help mobilize the community" (ID-KYG-HW-001).

"We were given the dates a bit early so it gave us time to go to the chief baraza and social gathering to inform them about the exercise. We also held meetings with stakeholders; this includes the ward reps, Imams, Pastors and representatives of Women groups. These helped us to spread the information to other people in the village" (IDI-KLN-HW-003).

A challenge presented in the area of mobilization and sensitization was a lack of information and proper sensitisation which led to some community members refusing to take the drugs. Some community members would accept the drugs but would not consume them. The CHEWs stated that:

"Yes, there were some challenges because there are some communities who still have myths about the drugs and others still asking why they are being given the drugs and they are still healthy. Others still think it is for family planning, although only few people had those problems", (IDI-KLN-HW-006).

"People are accepting them but then they don't take them because they don't have the necessary awareness" (IDI-KYG-HW-001).

The absence of some community members from their homes was also one of the challenges experienced during sensitization, mobilization and drug distribution. A CHEW from Kaloleni stated that:

"The time of the activity, the turn up depends on the season. People are busy during the rainy season. "Most of the people that did not take the drugs are those that are not at home at the time of distribution" (IDI-KLN-HW-001).
A suggestion during planning was that the mobilisation and sensitisation activity be carried out when the community members were not busy on their farms and during the school holidays. A CHEW from Kayafungo stated that:

"My suggestion is at least August-December season is the best because people are always through with the harvesting or during the holidays e.g., December when people are on holidays. Also, the sensitization period should be increased to a month so that everybody can get the information" (IDI-KYG-HW-003).

\section{Information, Education and Communication (IEC)}

Apart from awareness creation done through the door to door and using public address systems, the CHEWS were furnished with information education, communication materials such as flyers, booklets, posters and burners. They were instructed to display them in areas accessed frequently by community members such as market centres, mosques churches, schools and chiefs' camps. This was to ensure that the information on the IEC materials was accessible to the majority of community members. During the interviews, it was stated that:

"The materials are designed and produced by the NTD national office. We received posters, fliers, T-shirts and bags" (IDI-CNTD).

"We sensitized the community by telling them that we want to prevent $L F$, so we showed them pictures portraying the side effects of $L F$, pictures of people suffering from swollen genitals, swelling breast, swelling legs, you see, showing them the actual disease how it is like. So, we sensitize the community through the effects of the disease, swollen genitals and swollen limbs", (IDI-KYG-HW-001).

"Apart from creation of awareness which was done through public address, I had two burners that I placed where people can see them well so they can get more details of the activity then. I had some fliers that I distributed to the community, if someone sees those pictures that is enough information to make them accept the drugs. Health education was done through many

136 | This work is licensed under a Creative Commons Attribution 4.0 International License. 
ways; mobilization, burners and fliers" (IDKLN-HW-001).

Few IEC materials for distribution to the community members was a major challenge encountered during sensitization and mobilization. The SCNTDs coordinator stated that:

"A CDD was expected to have many fliers to distribute one in every household but they only received just one flier which they were using when visiting the households. Posters were very few. Say one per sub-location" (IDI-SC-NTD).

There were suggestions of involving the county in the production of the IEC materials to ensure that they were enough for distribution to all the households and key areas in the community. There were also various suggestions during sensitization such as increasing the sensitization period, recruitment of more CDDs, the inclusion of religious leaders to ensure improved uptake and delivery of MDA among the community members. The CNTDs coordinator and a CHEW from Kaloleni stated that:

"County to be given a chance to produce the IEC materials this will ensure enough fliers for CDDs to leave in the HH" (IDI-CNTD).

"I can suggest that the sensitization is for at least two weeks then followed with registration. The CDD number is to be increased. Enough sensitization will enable us to get out of those myths and misconceptions from the community, at least one month will be enough. Religious leaders should also be sensitized and also involves so that they can empower their followers" (IDI-KLN-HW-001).

\section{Record Keeping and Reporting}

The CHEWs were also assigned the role of record keeping. This included the household $(\mathrm{HH})$ register containing the number of members, their names, ages, residence, and number of drugs needed per household. This was generated by the community drug distributors on a door-to-door visit prior to the MDA. CHEWs from Kaloleni and Kayafungo stated that:
"The name of CDD, the date, amount of drugs given, the village, total of people administered total number of male and female, balance of drugs, the damaged drugs, and records of those people with signs of swollen limbs. We got only two" (IDI-KLN-HW-002).

There were challenges experienced during the registration of households. These were few CDDs, unavailability of members, myths, and misconceptions as stated by a CHEW from Kaloleni and one from Kayafungo.

"There were some households that were not registered and others registered but during the registration were not around, all this contributed to poor program performance. Those who missed registration was because there were few CDDs and the households were many" (IDIKLN-HW-001).

"Some households were not registered, and those who were registered some of them lack enough sensitization and also some have those myths and misconceptions and some household members were not available" (IDI-KYG-HW001).

There was also a reporting form for drug collection and a tally sheet for drug distribution. The tally sheet contained the number of people issued with drugs as per age, the number of drugs distributed, the number of drugs remaining, and the number of drugs spoilt. At the end of each distribution day, the CDDs summarised the data in each register and recorded it in the summary section at the end of the register. A CHEW from Kayafungo stated that:

"On the drugs, they were recording the starting balance, the top-ups, and the end balance including the drugs that got lost and those which were wasted. For example, when one takes the drug and vomits that a wasted drug" (IDIKYG-HW-002).

The poor network connection was one of the challenges cited during reporting. The CDDs at the end of each drug distribution day were required to take photos of the summarised tally sheets and send them using the internet to their respective CHEW. In areas, with poor network coverage, the CDDs were unable to do this and had to wait until the last 
day of drug distribution to manually take the tally sheets to the health workers. This led to delayed submission of reports. Another challenge was the time allocated for the drug distribution. There was not enough time for the CDDs to distribute the drugs and tally on a daily basis. The CNTDs coordinator stated that:

"There were challenges such as poor network. This delayed the submission of reports which required the CDDs to take photos of the summarised tally sheets and send them to the CHEWs. In an area that had challenges with the internet. They had to wait to the last day to submit reports personally" (IDI-CNTD).

One of the mitigation measures for delayed reporting was organizing for a central point where the CHEWs would collect the tally sheets from the CDDs. A CHEW from Kaloleni stated that:

\begin{abstract}
"The problem was transport for both CDDs and community health workers because bikes are few and the cash is given at the end of the activity and you have to pick the tallying sheets every day because the CDDs can't come to the office every day to bring them. I had to select a specific point where I can collect them. Also, we had a challenge during the follow up" (ID-KLN-HW001).
\end{abstract}

\section{DISCUSSION}

Mass drug administration, a strategy in which all individuals in a population are subjected to treatment without an individual diagnosis, has been recommended by the WHO for controlling and eliminating several NTDs, including LF. Many countries have implemented MDA for LF campaigns, successfully reducing the prevalence levels of the microfilariae in endemic populations and between the year 2000 and 2015, the WHO reported that more than 6.2 billion doses of treatment were administered to more than 830 million people in 64 endemic countries, reducing the transmission risk by 45\% [26]. Despite these positive global achievements in treatment provision, MDA for LF programmes continues to face numerous challenges that result in low treatment coverage levels and non-compliance by the communities [27-31]. Programmatic challenges include; sustaining timely distributions of drugs, establishing accurate monitoring and evaluation systems by the communities, increasing involvement of the local communities, and engaging in effective advocacy for continued MDA for LF support [7]. It has been noted that MDA for LF programmes seems to focus more on tablet distribution than on the major implementation questions such as health education, side effects, morbidity management, and logistics [32]. Similarly, there are also reports that problems of low compliance to MDA for LF are more providerinitiated than by individual recipients' perceptions and practices. Several reports have highlighted implementation challenges in MDA for LF programmes [33-36].

Mass drug administration, a strategy in which all individuals in a population are subjected to treatment without an individual diagnosis, has been recommended by the $\mathrm{WHO}$ for controlling and eliminating several NTDs, including LF. The current study sought to understand the role of the CHEWs and NTDs coordinators on the delivery of a community-wide MDA program for LF elimination, challenges faced, and suggestions for improved program performance. Results of the current study indicate that there exists a proper mechanism for the planning, coordination, and implementation of LF MDA activities. There are a series of activities right from the national up to the community level meant to ensure the smooth running of the program. However, there are various challenges that contribute to the poor program performance during the LF MDA.

Results of the current study results indicate that the length of the LF MDA period was not adequate to ensure comprehensive coverage of the villages. There were suggestions for increasing the MDA period. This is supported by findings from a previous study in Tanzania where the NTD control program based in Dar-es-Salaam decided on duration of three days for all contexts [37]. The CHEWs noted that the period allocated for the MDA was inadequate resulting in poor treatment coverage. In urban areas, there were too many houses to visit in three days while in rural areas, the distance between the houses was too far. This is in contrast to a study in India that showed that some doctors wanted to limit the MDA campaigns to one 
day in order to minimize the lower rates of compliance on subsequent days [38]. Results from the current study further show that people living in urban areas are difficult to reach during sensitisation and drug distribution. This is in line with a study in India where urban participation is more challenging due to transient populations in these areas, higher possibilities of absenteeism, low-risk perception, insufficient information, and communication among others [39].

There were also other challenges associated with drug distribution such as poor road networks in endemic populations, inadequate transport resources for supervisory teams, and remoteness of some communities which are also known to affected drug distribution and have also been reported in a study in Kenya. [40]. Another challenge was the timing of the MDA where it coincided with the dry rainy season where most of the community members who did not take the drugs, were busy on their farms' harvesting. There were suggestions of planning the MDA activity when the community members were not busy on their farms to ensure the availability of the people. This is in line with a study in India where Inappropriate distribution time was cited as negatively affecting coverage [41].

Results of the current study have further shown that mobilisation and sensitisation for LF MDA, was one of the roles for both the CHEWs and NTDs coordinators both at the county and sub-county level. It was the CHEWs' responsibility together with the CDDs to reach out to communities to discuss LF and underline the importance of collaboration between them and communities during MDA campaigns. They clearly defined and explained the roles and responsibilities of the various organizations involved in MDA and supported the community in the planning and implementation of the MDA campaign. This was a continuous process carried out before and during the LF MDA activity. The current study found that their active and sustained participation would be vital in the long-term success of the program. This has also been reported in a study in the Philippines, where health workers were found to be the major source of information about LF and MDA for community members [42].
Results of the current study have further shown that community participation was one of the challenges during mobilisation and sensitisation. The ratio of CDDs to the assigned households was one of the When the process of community involvement does not function very well, myths and rumours about the program become rife and tend to hamper implementation. [42, 43]. Community participation has been found to be one of the major challenges to the success of the MDA program. A lack of community participation hampers program implementation of all drug administration programs rather than only MDA for LF. The focus of community participation is to have communities direct and manage the recruitment of volunteers and strategies for drug distribution. This is expected to help communities own the programs and bridge the gap between them and the health system [43, 29].

Results from the current study have shown that there is a need to involve the county government in the production of the IEC materials so as to ensure that they were enough for distribution to all the households and key areas in the community. This is because it was easier to convince them when they saw the pictures in the fliers and banners. In India, the authors discovered that in communities where IEC activities had been better, communities understood the benefits of the program and were more aware of the side effects. In these Indian communities, coverage and compliance rates tended to be higher [46].

From the current study, there were also various suggestions for improved sensitization such as increasing the period, recruitment of more CDDs, the inclusion of religious leaders to ensure improved uptake and delivery of MDA among the community members. A study in Mali demonstrated that social mobilization efforts could help engender more interest among community members, by getting traditional and religious leaders involved in these campaigns. It demonstrated that the volunteers worked with village chiefs and community health workers to devise strategies to help organize the social mobilization campaign [44]. Another suggestion was the involvement of the county government in the production of the IEC materials so as to ensure that they were enough for distribution to all the households and key areas in the community. This is because it was easier to 
convince them when they saw the pictures in the fliers and banners. In India, the authors discovered that in communities where IEC activities had been better, communities understood the benefits of the program and were more aware of the side effects. In these Indian communities, coverage and compliance rates tended to be higher [27].

Results from the current study also showed that training was conducted by the NTDs coordinators and CHEWs. The training cascaded from the national level to the community level where the CDDs were trained by the HWs. The one day allocated for the training of CDDs was perceived as inadequate and hurried. This was because some health workers had to travel from far leading to a delayed starting and an early ending so as to ensure that there was enough time to travel back to their respective residences. This is in line with studies carried out in Kenya and Tanzania where the health workers cited the training duration as inadequate $[43,34]$. Reported training times of one day was inadequate and hurried to prepare CDDs with the knowledge of the disease and distribution needed to complete their task. Inadequate training leads to misinformation being passed on to the community about the MDA program, the cause of LF, how it is transmitted, and the benefits of taking the drugs. When training of CDDs was perceived as inadequate by community members they were more likely to refuse to take the tablets [27].

Findings from this study revealed that the role of the CHEWs was mostly supervision of the drug distribution and support CDDs with varying levels of engagement and has also been reported in studies done in Kenya and Tanzania [43,37]. In the Philippines and Sierra Leone, it was reported that when the health officer was present during campaigns coverage increased [42, 36]. Results from the current study also indicate that inadequate amount of time to carry out the supervision and the ratio of CHEWs versus the CDDs to be supervised as factors that contributed to poor supervision. This becomes even harder when MDAs are conducted during the rainy season in these poor remote areas. Longer campaigns would allow them to reach more households and adequate time to finish their reporting duties [36, 27,]. From this study, the involvement of the County in funding by providing vehicles to the program would go a long way in supporting the supervision process.

Data reported for LF programs are treatment coverage, which is generated from the community level by health volunteers, coverage surveys, and case identification reports for clinical follow-up of conditions such as hydrocele and elephantiasis. Treatment data are recorded at the household level by community health volunteers (CHVs), summarized at the community level, and submitted to the various supervising units [45]. The current study revealed that delays in the submission of reports was one of the challenges identified in the LF elimination program. This was due to poor network as the reports were to be submitted electronically. Data reported for LF programs are treatment coverage, which is generated from the community level by the community drug distributors, summarised at the community level, and submitted to the community health workers for onward submission to the various supervising units [46, 44]. Weak health systems in NTD endemic areas pose a challenge to the delivery of interventions at adequate levels to at-risk populations. To effectively reach target populations with NTD treatment, weak health systems challenges must be addressed by fixing specific weaknesses in the HS building blocks-service delivery, health workforce, information, medical products and technologies, financing, and leadership and governance [47].

\section{STUDY LIMITATIONS}

A study limitation that could have arisen in data collection was the introduction of recall bias during the interviews, by the study participants.

\section{CONCLUSIONS}

The results showed that there is a need to involve all the key stakeholders throughout all implementation stages so as to create ownership of the MDA program. Planning and implementing robust community mobilisation and sensitization process, having proper drug distribution and reporting mechanisms, will ensure the successful implementation of the LF MDA program. 


\section{AUTHOR CONTRIBUTIONS}

DWN conceived the study. CO contributed to the study design. DWN, CO, LWK, WPO, and BWK coordinated the data collection. LWK and BWK were responsible for transcription and DWN conducted the data analysis. BWK wrote the manuscript all authors contributed to the refinement and approved the final manuscript

\section{FUNDING}

This work received financial support from the Coalition for Operational Research on Neglected Tropical Diseases (COR-NTD) NTDSC 138D, which is funded at the Task Force for Global Health primarily by the Bill \& Melinda Gates Foundation, by the UK aid from the British government and by the United States Agency for International Development through its Neglected Tropical Diseases Program. The funders had no role in the design of this study, data collection, and analyses decision to publish or preparation of the manuscript.

\section{ACKNOWLEDGEMENTS}

The authors are grateful to Kilifi County and Kaloleni sub-county stakeholders for their technical support during the implementation of this study. We appreciate all the participants who agreed to be interviewed and the data collectors who conducted the interviews. This study has been published with the permission of the Director-General, KEMRI.

\section{REFERENCES}

[1] WHO. Lymphatic filariasis. 2019. Fact sheet. https://www.who.int/newsroom/fact-sheets/detail/lymphaticfilariasis (accessed Dec 30, 2019).

[2] World Health Organization. (2005). Global Programme to Eliminate Lymphatic Filariasis: annual report on lymphatic filariasis 2003 (No. WHO/CDS/CPE/CEE/2005.52).

[3] World Health Organization. (2010). Progress report 2000-2009 and strategic plan 20102020 of the global programme to eliminate lymphatic filariasis: halfway towards eliminating lymphatic filariasis (No. WHO/HTM/NTD/PCT/2010.6).

[4] World Health Organization. Ending the neglect to attain the Sustainable Development Goals: a road map for neglected tropical diseases 2021-2030. Geneva: World Health Organization; 2020.

[5] Ottesen, E. A. (2000). The global programme to eliminate lymphatic filariasis. Tropical Medicine \& International Health, 5(9), 591-594.

[6] Bockarie, M. J., Kelly-Hope, L. A., Rebollo, M., \& Molyneux, D. H. (2013). Preventive chemotherapy as a strategy for elimination of neglected tropical parasitic diseases: endgame challenges. Philosophical Transactions of the Royal Society B: Biological Sciences, 368(1623), 20120144.

[7] Kyelem, D., Biswas, G., Bockarie, M. J., Bradley, M. H., El-Setouhy, M., Fischer, P. U., \& Ottesen, E. A. (2008). Determinants of success in national programs to eliminate lymphatic filariasis: a perspective identifying essential elements and research needs. The American journal of tropical medicine and hygiene, 79(4), 480-484.

[8] World Health Organization. (2010). Monitoring the building blocks of health systems: a handbook of indicators and their measurement strategies. World Health Organization

[9] Marinucci, F., Manyazewal, T., Paterniti, A. D., Medina-Moreno, S., Wattleworth, M., Hagembe, J., \& Redfield, R. R. (2013). Impact of horizontal approach in vertical program: continuous quality improvement of malaria and tuberculosis diagnostic services at primary-level medical laboratories in the context of HIV care and treatment program in Ethiopia. The American Journal of Tropical Medicine and Hygiene, 88(3), 547-551.

[10] Swanson, R. C., Atun, R., Best, A., Betigeri, A., de Campos, F., Chunharas, S., ... \& Omaswa, F. (2015). Strengthening health 
systems in low-income countries by enhancing organizational capacities and improving institutions. Globalization and health, 11(1), 1-8.

[11] Goldberg, J., \& Bryant, M. (2012). Country ownership and capacity building: the next buzzwords in health systems strengthening or a truly new approach to development. BMC public health, 12(1), 531.

[12] Van Olmen, J., Marchal, B., Van Damme, W., Kegels, G., \& Hill, P. S. (2012). Health systems frameworks in their political context: framing divergent agendas. $B M C$ Public Health, 12(1), 774.

[13] Mounier-Jack, S., Griffiths, U. K., Closser, S., Burchett, H., \& Marchal, B. (2014). Measuring the health system's impact of disease control programmes: a critical reflection on the WHO building blocks framework. BMC public health, 14(1), 278.

[14] Senkubuge, F., Modisenyane, M., \& Bishaw, T. (2014). Strengthening health systems by health sector reforms. Global health action, 7(1), 23568.

[15] Obermann, K., Chanturidze, T., Richardson, E., Tanirbergenov, S., Shoranov, M., \& Nurgozhaev, A. (2016). Data for development in health: a case study and monitoring framework from Kazakhstan. BMJ Global Health, 1(1).

[16] Mutale, W., Bond, V., Mwanamwenge, M. T., Mlewa, S., Balabanova, D., Spicer, N., \& Ayles, H. (2013). Systems thinking in practice: the current status of the six WHO building blocks for health system strengthening in three BHOMA intervention districts of Zambia: a baseline qualitative study. BMC health services research, 13(1), 291.

[17] Fisher, J. R., \& de Mello, M. C. (2011). Using the World Health Organization's 4SFramework to Strengthen National Strategies, Policies and Services to Address Mental Health Problems in Adolescents in
Resource-Constrained

Settings. International journal of mental health systems, 5(1), 23.

[18] Njenga, S. M., Kanyi, H. M., Mutungi, F. M., Okoyo, C., Matendechero, H. S., Pullan, R. L., ... \& Won, K. Y. (2017). Assessment of lymphatic filariasis prior to re-starting mass drug administration campaigns in coastal Kenya. Parasites \& vectors, 10(1), 99.

[19] World Health Organization. (2017). Guideline: alternative mass drug administration regimens to eliminate lymphatic filariasis (No. WHO/HTM/NTD/PCT/2017.07). World Health Organization.

[20] Ministry of Health. (2006). Taking the Kenya essential package for health to the community: a strategy for the delivery of level one services. Reversing the TrendsThe Second National Health Sector Strategic Plan of Kenya, i-50.

[21] "The Report of the Interim Independent Boundaries Review Commission (IIBRC): Delimitation of Constituencies and Recommendations on Local Authority Electoral Units and Administrative Boundaries for Districts and Other Units" (PDF). IEBC. 2010. Retrieved 29 May 2020.

[22] "2019 Kenya Population and Housing Census Results". Kenya National Bureau of Statistics. Retrieved 29 May 2020.

[23] "Kilifi County Statistical Abstract 2015". Kenya National Bureau of Statistics. Retrieved 29 May 2020

[24] Creswell, J. W. (2013). Qualitative inquiry: Choosing among five approaches. Los Angeles, CA, 244.

[25] Johnson, R. B., \& Onwuegbuzie, A. J. (2010). Mixed research. $R B$ Johnson \& $L B$ Christensen, Educational research: Quantitative, qualitative, and mixed approaches, 439-459. 
[26] World Health Organization. (2017). Guideline: alternative mass drug administration regimens to eliminate lymphatic filariasis (No. WHO/HTM/NTD/PCT/2017.07). World Health Organization.

[27] Babu, B. V., \& Kar, S. K. (2004). Coverage, compliance and some operational issues of mass drug administration during the programme to eliminate lymphatic filariasis in Orissa, India. Tropical Medicine \& International Health, 9(6), 702-709.

[28] Gunawardena, S., Ismail, M., Bradley, M., \& Karunaweera, N. (2007). Factors influencing drug compliance in the mass drug administration programme against filariasis in the Western province of Sri Lanka. Transactions of the Royal Society of Tropical Medicine and Hygiene, 101(5), 445-453.

[29] Partono, F., \& Maizels, R. M. (1989). Towards a filariasis-free community: evaluation of filariasis control over an eleven-year period in Flores, Indonesia. Transactions of the Royal Society of Tropical Medicine and Hygiene, 83(6), 821-826.

[30] Vanamail, P., Ramaiah, K. D., Subramanian, S., Pani, S. P., Yuvaraj, J., \& Das, P. K. (2005). Pattern of community compliance with spaced, single-dose, mass administrations of diethylcarbamazine or ivermectin, for the elimination of lymphatic filariasis from rural areas of southern India. Annals of Tropical Medicine \& Parasitology, 99(3), 237-242.

[31] Cantey, P. T., Rout, J., Rao, G., Williamson, J., \& Fox, L. M. (2010). Increasing compliance with mass drug administration programs for lymphatic filariasis in India through education and lymphedema management programs. PLoS Negl Trop Dis, 4(6), e728.

[32] Mathieu E, et al. Factors associated with participation in a campaign of mass treatment against lymphatic filariasis, in
Leogane, Haiti. Ann Trop Med Parasitol. 2004; 98(7):703-14.

[33] Lahariya, C., \& Mishra, A. (2008). Strengthening of mass drug administration implementation is required to eliminate lymphatic filariasis from India: an evaluation study. $J$ Vector Borne Dis, 45(4), 313-20.

[34] Kisoka, W. J., Tersbøl, B. P., Meyrowitsch, D. W., Simonsen, P. E., \& Mushi, D. L. (2016). Community Members' perceptions of Mass Drug Administration for Control of Lymphatic Filariasis in Rural and Urban Tanzania. Journal of biosocial science, 48(1), 94-112.

[35] Hodges, M. H., Smith, S. J., Fussum, D., Koroma, J. B., Conteh, A., Sonnie, M., ... \& Zhang, Y. (2010). High coverage of mass drug administration for lymphatic filariasis in rural and non-rural settings in the Western Area, Sierra Leone. Parasites \& vectors, 3(1), 120.

[36] Hodges, M. H., Sonnie, M., Turay, H., Conteh, A., MacCarthy, F., \& Sesay, S. (2012). Maintaining effective mass drug administration for lymphatic filariasis through in-process monitoring in Sierra Leone. Parasites \& vectors, 5(1), 232.

[37] Kisoka, W. J., Simonsen, P. E., Malecela, M. N., Tersbøl, B. P., Mushi, D. L., \& Meyrowitsch, D. W. (2014). Factors influencing drug uptake during mass drug administration for control of lymphatic filariasis in rural and urban Tanzania. PLoS One, 9(10), e109316.

[38] Cantey, P. T., Rout, J., Rao, G., Williamson, J., \& Fox, L. M. (2010). Increasing compliance with mass drug administration programs for lymphatic filariasis in India through education and lymphedema management programs. PLoS Negl Trop Dis, 4(6), e728.

[39] Vanamail, P., Ramaiah, K. D., Subramanian, S., Pani, S. P., Yuvaraj, J., \& Das, P. K. (2005). Pattern of community compliance 
with spaced, single-dose, mass administrations of diethylcarbamazine or ivermectin, for the elimination of lymphatic filariasis from rural areas of southern India. Annals of Tropical Medicine \& Parasitology, 99(3), 237-242.

[40] Njomo, D. W., Mukoko, D. A., Nyamongo, N. K., \& Karanja, J. (2014). Increasing coverage in mass drug administration for lymphatic filariasis elimination in an urban setting: a study of Malindi Town, Kenya. PLoS One, 9(1), e83413.

[41] Vanamail, P., Ramaiah, K. D., Subramanian, S., Pani, S. P., Yuvaraj, J., \& Das, P. K. (2005). Pattern of community compliance with spaced, single-dose, mass administrations of diethylcarbamazine or ivermectin, for the elimination of lymphatic filariasis from rural areas of southern India. Annals of Tropical Medicine \& Parasitology, 99(3), 237-242.

[42] Amarillo, M. L. E., Belizario, V. Y., SadiangAbay, J. T., Sison, S. A. M., \& Dayag, A. M. S. (2008). Factors associated with the acceptance of mass drug administration for the elimination of lymphatic filariasis in Agusan del Sur, Philippines. Parasites \& Vectors, 1(1), 14.

[43] Njomo, D. W., Amuyunzu-Nyamongo, M., Magambo, J. K., Ngure, P. K., \& Njenga, S. M. (2012). Factors associated with the motivation of community drug distributors in the Lymphatic Filariasis Elimination Programme in Kenya. Southern African Journal of Epidemiology and Infection, 27(2), 66-70.

[44] Dembélé, M., Bamani, S., Dembélé, R., Traoré, M. O., Goita, S., Traoré, M. N., ... \& MacArthur, C. (2012). Implementing preventive chemotherapy through an integrated national neglected tropical disease control program in Mali. PLoS Negl Trop Dis, 6(3), e1574.

[45] da-Costa Vroom, F. B., Aryeetey, R., Boateng, R., Anto, F., Aikins, M., Gyapong, M., \& Gyapong, J. (2015). Data reporting constraints for the lymphatic filariasis mass drug administration activities in two districts in Ghana: A qualitative study. SAGE open medicine, 3 , 2050312115594083.

[46] Cavalli, A., Bamba, S. I., Traore, M. N., Boelaert, M., Coulibaly, Y., Polman, K. ... \& Van Dormael, M. (2010). Interactions between global health initiatives and country health systems: the case of a neglected tropical diseases control program in Mali. PLoS Negl Trop Dis, 4(8), e798.

[47] Nonvignon, J., Mensah, E., Vroom, F. B. D. C., Adjei, S., \& Gyapong, J. O. (2016). The role of health systems in the control of neglected tropical diseases in Sub-Saharan Africa. In Neglected Tropical DiseasesSub-Saharan Africa (pp. 385-405). Springer, Cham. 